\title{
The formation of sporadic $E$ layers by a vortical perturbation excited in a horizontal wind shear flow
}

\author{
G. G. Didebulidze and L. N. Lomidze \\ Georgian National Astrophysical Observatory, Ilia Chavchavadze State University, A. Kazbegi Ave. 2a, Tbilisi 0160, Georgia
}

Received: 8 May 2007 - Revised: 7 April 2008 - Accepted: 6 May 2008 - Published: 24 June 2008

\begin{abstract}
The formation of the mid-latitude sporadic E layers ( $E_{S}$ layers) by an atmospheric vortical perturbation excited in a horizontal shear flow (horizontal wind with a horizontal linear shear) is investigated. A three-dimensional atmospheric vortical perturbation (atmospheric shear waves), whose velocity vector is in the horizontal plane and has a vertical wavenumber $k_{z} \neq 0$, can provide a vertical shear of the horizontal wind. The shear waves influence the vertical transport of heavy metallic ions and their convergence into thin and dense horizontal layers. The proposed mechanism takes into account the dynamical influence of the shear wave velocity in the horizontal wind on the vertical drift velocity of the ions. It also can explain the multi-layer structure of $E_{s}$ layers. The pattern of the multi-layer structure depends on the value of the shear-wave vertical wavelength, the ion-neutral collision frequency and the direction of the background horizontal wind. The modelling of formation of sporadic E layers with a single and a double peak is presented. Also, the importance of shear wave coupling with short-period atmospheric gravity waves (AGWs) on the variations of sporadic $\mathrm{E}$ layer ion density is examined and discussed.
\end{abstract}

Keywords. Ionosphere (Ionospheric irregularities; Midlatitude ionosphere) - Meteorology and atmospheric dynamics (Waves and tides)

\section{Introduction}

The formation of the mid-latitude sporadic E layers ( $E_{s}$ layers) and their temporal evolution results from the action of various physico-chemical processes in the mesosphere-lower thermosphere. A vertical shear in the horizontal neutral wind is the major cause of mid-latitude $E_{s}$ layer formation (e.g.

Correspondence to: G. G. Didebulidze

(didebulidze@genao.org) see Whitehead, 1989, and references therein). Tidal motions and atmospheric waves play the key role in this formation process by providing the vertical wind shears needed for the ions to converge into thin and dense horizontal layers (Mathews, 1998; Haldoupis et al., 2006). In addition to the diurnal and semidiurnal tides, which are present regularly in the lower thermosphere, the action of planetary waves (PWs) and atmospheric gravity waves (AGWs) may influence the formation of sporadic E layers as well (Chimonas, 1971; Haldoupis and Pancheva, 2002; Shalimov and Haldoupis, 2002; Pancheva et al., 2003). In a proposed interpretation, this influence was attributed to the accumulation of metallic ions caused by the action of horizontal PW - and AGW - related wind shear.

Several observations on the vertical variability of the sporadic $E$ layers, such as the appearance of multi-layered structures, underline the importance of their relation to the atmospheric waves and the horizontally inhomogeneous wind (e.g. see Larsen et al., 2005). These observed features of $E_{S}$ layers were considered by many authors as a result of an interaction of AGWs propagating through the region (Mathews et al., 1993; Yokoyama et al., 2003, 2004; Larsen et al., 2005). This emphasized the necessity of investigation of the $E_{s}$ formation process in relation with the variability in the horizontal wind (e.g. see more details in Kelley et al., 1995; Hussey et al., 1997; Yokoyama et al., 2003, 2005; Larsen et al., 2005).

In the present study, the possible effects on the formation of mid-latitude sporadic E layers in relation with a horizontal shear flow (horizontal wind with a horizontal linear shear) will be investigated. In this case the major cause of providing the vertical wind shear in the horizontal wind, which is needed for vertical ion convergence, is a horizontal shear flow exciting a three-dimensional vortical perturbation, that is referred to as "atmospheric shear wave" (Didebulidze, 1999; Didebulidze et al., 2004). The shear wave influences the vertical transport of heavy metallic ions and

Published by Copernicus Publications on behalf of the European Geosciences Union. 
their convergence into thin layers in a similar way as in the formation mechanism of a vertical wind shear in the horizontal wind (e.g. see Whitehead, 1989, and references therein). In the framework of the mechanism, proposed in the present work, the appearance of $E_{s}$ layers with several peaks will be simulated and explained. Also it will be shown that the shear wave property, with regards to its transformation to shortperiod atmospheric gravity waves, can influence the variability of sporadic E layers.

\section{Ion vertical drift velocity in the horizontal shear flow}

The physics of the sporadic E layer formation is based on the "windshear" theory, according to which a vertical shear in the horizontal neutral wind can cause, by the combined action of the ion-neutral collisional coupling and the geomagnetic Lorentz forcing, the long-lived metallic ions to move vertically and accumulate into thin and dense plasma layers. The process is governed by ion dynamics, with the magnetized electrons only following the ions to maintain charge neutrality (Whitehead, 1989, and references therein). A similar mechanism of accumulation of ions is possible in the case of a three-dimensional vortical perturbation as shown by Didebulidze (1999) and Didebulidze et al. (2004). The variation in the shear wave horizontal velocity with height can accumulate ions at some height which depends on the vertical wavenumber. The equation describing this process is derived from the steady-state ion momentum equation where the effects of diffusion and electric forces are neglected (MacLeod, 1966):

$M v_{i n}\left(\boldsymbol{v}_{\boldsymbol{i}}-\boldsymbol{v}_{\boldsymbol{n}}\right)-e \cdot \boldsymbol{v}_{\boldsymbol{i}} \times \boldsymbol{B}=0$,

where $M$ is the ion mass, $v_{i n}$ is the ion-neutral collision frequency, $\boldsymbol{B}$ is the magnetic field vector; $\boldsymbol{v}_{\boldsymbol{i}}$ and $\boldsymbol{v}_{\boldsymbol{n}}$ are the velocities of ions and neutral particles, respectively.

In the present consideration, the velocity of neutral particles $\boldsymbol{v}_{\boldsymbol{n}}$ is equal to the sum of background horizontal wind $\left(\boldsymbol{u}_{0}\left(u_{0}, 0,0\right)\right)$ and the wavelike perturbation $(\boldsymbol{v})$ velocities

$v_{n}=u_{0}+v$.

We take a right-handed set of coordinates $(x, y, z)$ with $x$ directed to background horizontal wind and $z$ vertically upwards. In Eq. (2), $\boldsymbol{v}=\boldsymbol{v}(u, v, w)$ is assumed to represent the atmospheric wave velocity and $u, v$ and $w$ are its components in the $\mathrm{x}-, \mathrm{y}$ - and $\mathrm{z}$-directions, respectively. Such waves, in addition with the background neutral horizontal wind, can force a vertical transport of charged particles and thus form a sporadic E layer. From Eqs. (1) and (2) we can easily obtain the expression for the ion vertical velocity in the following form:

$w_{i}=$

$\frac{-\cos I(\sin I \cos \delta+\kappa \sin \delta)\left(u+u_{0}\right)+\cos I(\sin I \sin \delta-\kappa \cos \delta) v+\left(\kappa^{2}+\sin ^{2} I\right) w}{1+\kappa^{2}}$, where $\kappa=\frac{v_{i n}}{\omega_{i}}, \omega_{i}=\frac{e B}{M}$ is the ion gyrofrequency, $I$ is the magnetic dip angle, $\delta$ is the angle (positive counterclockwise from north) between magnetic north and the horizontal wind velocity $\boldsymbol{u}_{\boldsymbol{o}}$. In the case of $\delta=0$, the $\mathrm{x}$ - and y-axes are oriented in the magnetic north and west directions, respectively. In the present consideration, the main process of sporadic $\mathrm{E}$ layer formation is the vertical transport of ions. The variability of the ion velocity horizontal components is assumed to be much smaller than that of the vertical component and therefore is omitted.

Equation (3) shows that the ion vertical velocity (vertical drift velocity) $w_{i}$ is determined by wavelike perturbations in the background horizontal wind in the same (first term in numerator), its perpendicular (second term) and the vertical (third term) directions. For the considered regions of the mid-latitude lower thermosphere the variations of these parameters, the ion-neutral collision frequency $v_{i n}$ (or $\kappa$ ), the geometry of the magnetic field, and the background horizontal wind, all influence the ion vertical motion. Equation (3) shows that in the north-hemisphere mid-latitudes, where $\cos I(\sin I \cos \delta+\kappa \sin \delta)>0$, and in the presence of a horizontal wind with a vertical shear, the ion vertical drift velocity $w_{i} \propto-u_{0}$. In this case the ions convergence point $w_{i}=0$ occurrs at heights where the background neutral horizontal wind velocity $u_{0}=0$. A horizontal wind with a vertical shear causes downward motion of ions $\left(w_{i}<0\right)$ for height above the ion convergence node (where $u_{0}>0$ ), and upward motion $\left(w_{i}>0\right)$ below this point (where $u_{0}<0$ ), thus ions accumulate into thin horizontal layers close to their convergence node height. If $\cos I(\sin I \cos \delta+\kappa \sin \delta)<0$, then the ion vertical motion is similar to that for the opposite direction of the horizontal wind considered above. These processes are typical for the classical "vertical windshear" mechanism of $E_{S}$ formation in the meridional (for height $>125 \mathrm{~km}$ where $\kappa<1$ ) and zonal winds (for height $<125 \mathrm{~km}$ where $\kappa>1$ ), as described by many authors (Whitehead, 1989, and references therein; Mathews, 1998).

In the presence of a height independent background horizontal wind $\left(\boldsymbol{u}_{0}\left(u_{0}, 0,0\right)\right)$, a vertical shear in the neutral wind $\boldsymbol{v}_{\boldsymbol{n}}$ in Eq. (2), which is necessary for ion vertical convergence and thus sporadic $\mathrm{E}$ layer formation, can be provided by atmospheric waves whose magnitude (velocity $\boldsymbol{v}$ ) varies with height. Such vertical shear can appear in the magnitude of atmospheric waves excited in a horizontal shear flow

$u_{0}=a y$.

Here $a$ is the linear shear in the horizontal wind. As shown by Didebulidze (1999), Didebulidze et al. (2004), the excitation of acoustic-gravity waves and vortical perturbations is possible, as described by the horizontal shear flow Eq. (4). A three-dimensional atmospheric vortical perturbation (atmospheric shear wave), whose velocity vector $(v)$ is in the horizontal plane $(u, v \neq 0$ and $w=0)$ and varying with height, can provide a vertical shear in the horizontal wind. The shear waves can influence the vertical transport of ions and 
their convergence into thin horizontal layers in a way similar to the well known windshear theory (e.g. see Whitehead, 1989). Equation (3) also shows that the different evolution of $u$ and $v$ components of shear wave velocity and its transformation into short-period AGWs (with $w \neq 0$ ) can influence the ion vertical drift velocity. This effect of shear waves on this drift velocity $w_{i}$ also depends on the ion-neutral collision frequency $v_{i n}$ (or $\kappa$ ) and the angle $\delta$ between the directions of magnetic north and the background flow, which in turn affects the sporadic E electron density. The properties of vortical perturbation evolving in the horizontal shear flow, necessary for the formation of sporadic E, are considered below in more detail.

\section{Atmospheric shear waves}

The horizontal wind in the upper atmosphere is mainly inhomogeneous. The value and direction of the background horizontal wind undergoes daily, seasonal, inter-seasonal, etc., variations. In the lower thermosphere the tidal motion, planetary waves and AGWs are considered as one of the important factors, which affect neutral wind and charged particle densities. In our study $\boldsymbol{v}=\boldsymbol{v}(u, v, w)$ represents the atmospheric gravity, or shear, wave velocity evolving in a horizontal shear flow of Eq. (4), having characteristic periods smaller than those of tides and planetary waves. Internal waves in an inviscid isothermal atmosphere, when the gravity force and the background horizontal wind $\left(\boldsymbol{u}_{0}\right)$ are taken into account, are described by the following, well known, set of the linearized (continuity, motion and adiabatic) equations:

$$
\begin{aligned}
& \rho_{0}\left[\frac{\partial \boldsymbol{v}}{\partial t}+\left(\boldsymbol{u}_{\mathbf{0}} \cdot \nabla\right) \boldsymbol{v}+(\boldsymbol{v} \cdot \nabla) \boldsymbol{u}_{\mathbf{0}}\right]=-\nabla p+\rho \boldsymbol{g}, \\
& \frac{\partial \rho}{\partial t}+\left(\boldsymbol{u}_{\mathbf{0}} \cdot \nabla\right) \rho=-\boldsymbol{v} \cdot \nabla \rho-\rho_{0}(\nabla \cdot \boldsymbol{v}), \\
& \frac{\partial p}{\partial t}+\left(\boldsymbol{u}_{\mathbf{0}} \cdot \nabla\right) p=-c_{s}^{2} \rho_{0}(\nabla \cdot \boldsymbol{v})-\boldsymbol{v} \cdot \nabla p_{0},
\end{aligned}
$$

where $p$ and $\rho$ are perturbed pressure and density, $\boldsymbol{g}$ is the acceleration due to gravity, $c_{s}=\left(\gamma p_{0} / \rho_{0}\right)^{1 / 2}$ is the speed of sound, $\gamma$ is the ratio of the specific heats $(\gamma=1.4)$; $p_{0}=p_{00} \exp (-z / H)$ and $\rho_{0}=\rho_{00} \exp (-z / H)$ are the unperturbed barometric height profiles for pressure and density, respectively; $z=h-h_{0}$ is the difference between an actual and some initial height $h_{0}$ and $H=c_{s}^{2} / \gamma g$ is the atmospheric (neutral gas) scale height. Note that in Eq. (5) we assume conditions of hydrostatic equilibrium, that is $\rho_{0} g-\nabla p_{o}=0$.

We transform the linear perturbations of atmospheric parameters by the following form (Hines, 1960; Didebulidze, 1999):

$$
\begin{aligned}
& p, \rho(x, y, z, t)=p_{1}, \rho_{1}(x, y, z, t) \exp \left(-\frac{z}{2 H}\right) \\
& u, v, w(x, y, z, t)=u_{1}, v_{1}, w_{1}(x, y, z, t) \exp \left(\frac{z}{2 H}\right)
\end{aligned}
$$

$q_{1}=\int_{-\infty}^{+\infty} q_{k}(t) \exp \left[i\left(k_{x} x+k_{t}(t) y+k_{z} z\right)\right] d k_{x} d k_{y} d k_{z}$

where

$$
q_{1} \equiv\left\{p_{1}, \rho_{1}, u_{1}, v_{1}, w_{1}\right\}
$$

and $q_{k}(t) \equiv\left\{p_{k}, \rho_{k}, u_{k}, v_{k}, w_{k}\right\}(t)$ are the vectors of perturbed values and amplitudes of their spatial Fourier harmonics $(\mathrm{SFH}), \quad$ respectively. $\quad k_{t}(t)=k_{y}-a k_{x} t$, $\boldsymbol{k}(t)=\boldsymbol{k}\left(k_{x}, k_{t}(t), k_{z}\right)$ is the time variant wavenumber. By use of the transformation (8-10) in Eqs. (5-7) we get the following set of the linearized equations:

$$
\begin{aligned}
& \rho_{00} u_{k}^{\prime}=-i k_{x} p_{k}-a \rho_{00} v_{k}, \\
& \rho_{00} v_{k}^{\prime}=-i k_{t}(t) p_{k}, \\
& \rho_{00} w_{k}^{\prime}=-\left(i k_{z}-\frac{1}{2 H}\right) p_{k}-g \rho_{k}, \\
& \frac{\rho_{k}^{\prime}}{\rho_{00}}=-i\left(k_{x} u_{k}+k_{t}(t) v_{k}\right)-\left(i k_{z}-\frac{1}{2 H}\right) w_{k}, \\
& \frac{p_{k}^{\prime}}{\rho_{00} c_{s}^{2}}=-i\left(k_{x} u_{k}+k_{t}(t) v_{k}\right)-\left(i k_{z}-\varepsilon\right) w_{k},
\end{aligned}
$$

where prime denotes a time derivative and $\varepsilon=(2-\gamma) / 2 \gamma H$ is the isothermal Eckart coefficient (Gossard and Hooke, 1975).

In the governing Eqs. (5-7), the spatial inhomogeneity is caused by horizontal winds $\boldsymbol{u}_{0}\left[u_{0}(y), 0,0\right]$, unperturbed density $\rho_{0}(z)$ and pressure $p_{0}(z)$. Transformation of Eqs. (8-10) allows us to reduce these equations to Eqs. (11-15), where the inhomogeneity $\left(k_{t}\right)$ occurs in time. Thus we have reduced Eqs. (5-7) to an initial-value problem.

If we reduce the set of Eqs. (11-15) to the equation for $p_{k}$ and search for the spectrum of atmospheric waves with time varying frequencies $\omega(t)$ (for $a^{2} \ll \omega_{b}^{2}$ ), we obtain the following acoustic-gravity and shear wave characteristic frequencies (Didebulidze et al., 2004):

$$
\begin{aligned}
& \omega_{a, g}^{2}(t)=\frac{1}{2} c_{s}^{2}\left(|\boldsymbol{k}(t)|^{2}+\frac{1}{4 H^{2}}\right) \\
& \pm \sqrt{\frac{1}{4} c_{s}^{4}\left(|\boldsymbol{k}(t)|^{2}+\frac{1}{4 H^{2}}\right)^{2}-\omega_{b}^{2} c_{s}^{2}\left[\left(1+\frac{10}{R_{h}}\right) k_{x}^{2}+k_{t}^{2}(t)\right]} \\
& \omega_{s h}=0,
\end{aligned}
$$

where $\left.\omega_{b}=[(\gamma-1) g) /(\gamma H)\right]^{1 / 2}$ is the isothermal BruntVäisälä frequency, $R_{h}=\omega_{b}^{2} / a^{2}$ is the measure of the relative strength of stratification and the shear of the background flow (an analogy of the Richardson number for a horizontal shear flow). In Eq. (16) the high $\omega_{a}(t)$ (upper sign "+") and low $\omega_{g}(t)$ (lower sign "-") represent the frequencies of atmospheric acoustic and gravity waves evolving in a horizontal shear flow of Eq. (3), respectively. In the case of no shear $(a=0)$, Eq. (16) leads to the spectrum of acoustic-gravity 
waves obtained by Hines (1960). Equations (11-17), with regards to acoustic-gravity waves, also leads to the evolution of a shear wave $\left(\omega_{s h}=0\right)$, whose excitation is due to the presence of a horizontal shear flow. For $R_{h} \gg 10$, i.e. when the shear is very small, the solution for the shear wave time variant spatial amplitude, according to Didebulidze (1999) and Didebulidze et al. (2004), has the following form:

$u_{k}(t)=-\frac{A_{s h}\left(k_{z}-\frac{i}{2 H}\right)\left(k_{x}^{2}+k_{y}^{2}\right)^{2}}{k_{x}^{2}\left(k_{z}^{2}+\frac{1}{4 H^{2}}\right)} \frac{k_{t}}{k_{x}^{2}+k_{t}^{2}}$,

$v_{k}(t)=\frac{A_{s h}\left(k_{z}-\frac{i}{2 H}\right)\left(k_{x}^{2}+k_{y}^{2}\right)^{2}}{k_{x}\left(k_{z}^{2}+\frac{1}{4 H^{2}}\right)} \frac{1}{k_{x}^{2}+k_{t}^{2}}$,

$w_{k}(t)=0$.

where $A_{s h}$ takes a value, which we can chose independently from the wavenumbers for characteristic shear waves SFH amplitudes. Note, that the value $2 a \rho_{00} A_{s h} / g$ is the SFH amplitude of density perturbation for the shear wave (Didebulidze et al., 2004).

The shear wave corresponds to the standing type of an incompressible $(\nabla \cdot v=0)$ vortical perturbation $\left(\left(\nabla \times \boldsymbol{v}_{k}\right)_{z}=\right.$ const where $\left.\boldsymbol{v}_{k}=\left(u_{k}, v_{k}, w_{k}\right)\right)$ excited in the horizontal shear flow. According to Eqs. (18) and (19), the changes in the shear wave velocity amplitude are essential at $t \leq t_{a}\left(t_{a}=2\left|\frac{k_{y}}{a k_{x}}\right|\right)$, whereas for $t>t_{a}$ the acoustic wave frequency $\omega_{a}(t)$ continuously increases and the gravity wave frequency $\omega_{g}(t)$ tends to the isothermal Brunt-Väisälä frequency $\omega_{b}$.

Equations (18-20) show that the shear wave energy (spectral energy), which is mainly determined by its kinetic energy $\propto \rho_{00} \frac{U_{k}^{2}+V_{k}^{2}}{2}$ (Didebulidze, 1999), increase up to its maximum value at $t=t_{a} / 2$. Here $U_{k}(t)=\operatorname{Re}\left[u_{k}(t)\right]$ and $V_{k}(t)=\operatorname{Re}\left[v_{k}(t)\right]$. The shear wave acquires energy from the shear flow for $t<t_{a} / 2$. For $t>t_{a} / 2$ the shear wave gives energy back to the background flow or transforms into AGWs.

Such three-dimensional vortical-type perturbation (shear wave) excited in a horizontal shear flow of Eq. (4) has mainly a horizontal velocity, which according to Eqs. (8-10) and Eqs. (18-20) varies with height. In the case of $\lambda_{x}, \lambda_{y} \gg \lambda_{z}$ (or $\left|k_{x}\right|, \quad\left|k_{y}\right|, \quad\left|k_{t}\right| \ll\left|k_{z}\right|$ for $t<t_{a}$ ) and $a^{2} \ll \omega_{b}^{2}$ (or $R_{h} \gg 10$ ), the change of the shear wave velocity is smaller in the horizontal direction than in the vertical direction. Here $\lambda_{x}=\frac{2 \pi}{\left|k_{x}\right|}, \lambda_{y}=\frac{2 \pi}{\left|k_{y}\right|}$ and $\lambda_{z}=\frac{2 \pi}{\left|k_{z}\right|}$ are wavelengths in $\mathrm{x}-, \mathrm{y}-$ and z-directions, respectively.

For some heights of the lower thermosphere with width of about half of vertical wavelength $\lambda_{z} / 2$, where the shear wave velocity $(\boldsymbol{v})$ changes in direction, this wave can cause the ions move downwards $\left(w_{i}<0\right)$ and upward $\left(w_{i}>0\right)$ thus they can converge into a thin layer at heights where $w_{i}=0$, similarly as in the formation of sporadic $\mathrm{E}$ layers by a vertical windshear in the horizontal wind (Whitehead, 1989, and references therein). There can be a few such regions of lower thermospheric heights where heavy metalic ions can be forced to form multiple layers (e.g. see Turunen et al., 1993; Hocke et al., 2001; Damtie et al., 2002; Wakabayashi and Ono, 2005, and references therein; Haldoupis et al., 2006).

The set of Eqs. (11-15) describes the evolution of a threedimensional vortical type perturbation in a horizontal shear flow (4) taking into account its coupling with acoustic and gravity waves of Eq. (16) (Didebulidze et al., 2004). The expressions (18-20) of SFH amplitudes do not include changes caused by shear wave coupling with acoustic and gravity waves of Eq. (16) in a horizontal shear flow of Eq. (4). Important changes of shear wave amplitudes, caused by AGWs, occur for horizontal wavelengths comparable to atmospheric scale height, $\lambda_{x}, \lambda_{y}=O(H)$, or/and for the greater values of shear $a$ (i.e. $R_{h}=O(10)$ ).

Next we use wavelengths $\lambda_{x}, \lambda_{y} \gg \lambda_{z}$ and smaller value of shear $a$ (i.e. $R_{h} \gg 10$ ) when for $t<t_{a}$ the SFH amplitudes of shear wave velocity components, $u_{k}(t), v_{k}(t)$ and $w_{k}(t)$, are close to their independent evolution described by Eqs. (18-20). In the following we investigate the formation of sporadic E layers under the influence of shear waves numerically, by considering the evolving shear wave properties for different velocity amplitudes in the horizontal x- and ydirections, as well as coupling with AGWs. The latter will appear as AGW-like oscillations in $u_{k}(t), v_{k}(t)$ and $w_{k}(t)$ with frequency of Eq. (16).

\section{Formation of sporadic E layers in the horizontal shear flow}

Now we consider the ion velocity $w_{i}(z, t)$, Eq. (3), and compute its height variations under the influence of an atmospheric vortical type perturbation evolving in the horizontal shear flow of Eq. (4). We use analytical, Eqs. (18-20), and numerical solutions of Eqs. (11-15) for amplitudes of shear waves velocity SFH. The presence of a shear wave is considered at a given wavenumber. In this case according to Eqs. (9) and (10) the shear wave velocity components take the following form:

$$
\begin{aligned}
& u(x, y, z, t)=e^{z} \not 2 H \cdot \operatorname{Re}\left\{u_{k}(t) \exp [i \phi(x, y, z, t)]\right\}, \\
& v(x, y, z, t)=e^{z} / 2 H \cdot \operatorname{Re}\left\{v_{k}(t) \exp [i \phi(x, y, z, t)]\right\}, \\
& w(x, y, z, t)=e^{z} / 2 H \cdot \operatorname{Re}\left\{w_{k}(t) \exp [i \phi(x, y, z, t)]\right\},
\end{aligned}
$$

where $\phi(x, y, z ; t)=k_{x} x+k_{t} y+k_{z} z$.

By using Eqs. (21-23), we investigate the height profile variations of the ion vertical velocity $w_{i}(z, t)$, Eq. (3), for a given point $\left(x=x_{0}, y=y_{0}\right)$ in the horizontal plane, for the horizontal shear flow of Eq. (4) and the shear waves excited in this flow. Here we will use the analytic approach 
of Eqs. (18-20) and the numerical solutions of Eqs. (11-15) for shear wave SFH amplitudes, in order to estimate the velocities $u(z, t), v(z, t)$ and $w(z, t)$ given in Eqs. (21-23).

We investigate the formation of sporadic $\mathrm{E}$ in the midlatitude thermosphere region of $90-120 \mathrm{~km}$. Here we use a magnetic dip angle $I=60^{\circ}$ and the IGRF geomagnetic field model. The dominant ions are assumed to be $\mathrm{Fe}+(56 \mathrm{amu})$, the ion gyrofrequency $\omega_{i} \approx 80 \mathrm{~s}^{-1}$, whereas the ion-neutral collision frequency is computed from $v_{i n}=\left(2.62\left[\mathrm{~N}_{2}\right]+2.61\left[\mathrm{O}_{2}\right]+1.43[\mathrm{O}]\right) \cdot 10^{-10} \mathrm{~s}^{-1}$ (e.g. see Banks and Kockarts, 1973), where the neutral particle densities $\left[\mathrm{N}_{2}\right],\left[\mathrm{O}_{2}\right]$ and $[\mathrm{O}]\left(\mathrm{cm}^{-3}\right)$ are taken from the MSISE-90 atmospheric model (Hedin, 1991). The suggested mechanism of sporadic $\mathrm{E}$ formation is possible to be used for different seasons of year and solar phases. For the clearness of further calculations we consider nighttime conditions at solar minimum during a magnetically quiet summer-time period. Note that the present consideration may be developed for various compositions of ions including $\mathrm{Fe}+$ and $\mathrm{NO}+$ where the latter is the major ionic component at altitudes under consideration.

To demonstrate the formation of sporadic $\mathrm{E}$ layers under the influence of shear waves at a height of 90-120 km, we use below the numerical solution of the continuity equation for ions, taking into account the ion vertical velocity described by Eq. (3). In this case when $\lambda_{x}, \lambda_{y} \gg \lambda_{z}$, the horizontal ion transport is comparatively small and is omitted. Next, we assume quasineutrality in this region of the thermosphere and the Gaussian distribution of ions and electrons at an initial moment. Here the ion production and loss are considered comparatively small during the formation of sporadic E layers, and the temporal development of sporadic $\mathrm{E}$ is simulated only by its electron density as a function of height and time.

Figure 1 is as follows: panel (a) shows the numerical results of the temporal development of the spatial amplitudes of shear wave velocity (at $\left.h=h_{0}=82 \mathrm{~km}\right) \quad U_{k}(t)=\operatorname{Re}\left[u_{k}(t)\right]$ (dark dashed line - analytical approach, dark solid line numerical simulation), $V_{k}(t)=\operatorname{Re}\left[v_{k}(t)\right]$ (dashed line - analytical approach, solid line - numerical simulation) and $W_{k}(t)=\operatorname{Re}\left[w_{k}(t)\right]$ (dash-dotted line). Panel (b) shows the height variations of horizontal $u(h, t)$ (dashed line), $v(h, t)$ (solid line) and vertical $w(h, t)$ (dotted line) components of shear wave velocity. Panel (c) shows the height variations of the ion vertical drift velocity $w_{i}(h, t)$, whereas panel (d) shows the formation of a sporadic E layer (the electron density is normalized on its maximum value at the initial time) for time intervals: $t=0,5,10,15,20,25$ and $30 \mathrm{~min}$, in the horizontal shear flow. Here, the linear shear $a=10^{-4} \mathrm{~s}^{-1}$ and the shear wave horizontal and vertical wavelengths $\lambda_{x}=\lambda_{y}=754 \mathrm{~km}$ and $\lambda_{z}=25 \mathrm{~km}$, are used respectively. The initial conditions for the shear wave SFH amplitudes were chosen in accordance with Eqs. (18-20), for $A_{s h}=579 \mathrm{~m} / \mathrm{s}$. The horizontal wind was taken to be oriented to the magnetic north $(\delta=0)$, while the electron density peak height at the initial Gaussian distribution is $105 \mathrm{~km}$ and its half-width is $15 \mathrm{~km}$. For simplicity $x_{0}=0$ and $y_{0}=0$ are assumed.

Using the numerical solution for shear waves in Eq. (3) it leads to the ion vertical velocity (see Fig. 1c) for which ion convergence occurs at $h \approx 109 \mathrm{~km}$, at $t=5 \mathrm{~min}$, and then descends with time down to $h \approx 106 \mathrm{~km}$ at $t=30 \mathrm{~min}$, staying close to convergence node $\left(w_{i}=0\right)$. In this case the increase in the layer electron/ion density at $t=30 \mathrm{~min}$ is about 10 times that of the initial peak value. The consideration of the temporal evolution of a sporadic $\mathrm{E}$ for times $t-t_{0}>30 \mathrm{~min}$ needs the inclusion of ambipolar diffusion in the continuity equation of ions/electrons, which may stop the sporadic E density growth. In Fig. 1a the numerical solution for the shear wave velocity SFH amplitudes (for $t<t_{a}$ ) is close to the analytical description of its independent evolution. This evolution (Fig. 1a) is given for a time greater than that necessary for the formation of sporadic E layers (Fig. 1d). In this case it is noticeable that the shear wave evolution is close to the independent one $\left(t<t_{a}\right)$, described by Eqs. (18) and (20), and then transforms into AGWs $\left(t>t_{a}\right)$, with frequencies given by Eq. (16).

As noted in the previous subsection, the formation of multi-layered structures in sporadic $\mathrm{E}$ under the influence of a shear wave, is expected to take place for shorter vertical wavelengths $\lambda_{z}$. Figure 2 is as follows: panel (a) shows the temporal development (at $h=h_{0}=94 \mathrm{~km}$ ) of spatial amplitudes $U_{k}(t)$ of the shear wave velocity (dark dashed line analytical approach, dark solid line - numerical simulation), $V_{k}(t)$ (dashed line - analytical approach, solid line - numerical simulation) and $W_{k}(t)$ (dash-dotted line). Panel (b) shows the height variations of horizontal $u(h, t)$ (dashed line), $v(h, t)$ (solid line) and vertical $w(h, t)$ (dotted line) components of the shear wave velocity. Panel (c) shows the height variations of the ion vertical drift velocity $w_{i}(h, t)$, whereas panel (d) the formation of a sporadic $E$ layer (for electron density) for the time intervals: $t=0,5,10,15,20$, 25 and $30 \mathrm{~min}$ in the horizontal shear flow. Here the Gaussian peak height and its half-width at the initial moment are equal to $110 \mathrm{~km}$ and $10 \mathrm{~km}$, respectively, the shear wave vertical wavelength is $\lambda_{z}=8 \mathrm{~km}$ and $A_{s h}=1658 \mathrm{~m} / \mathrm{s}$. The values of the horizontal wind shear and the horizontal wavelengths are the same as in Fig. 1.

To obtain the formation of sporadic E layers with two main peaks (Fig. 2d) the background horizontal wind $\left(\boldsymbol{u}_{\boldsymbol{o}}\right)$ is taken to have a north-westward direction, $\delta=17^{\circ}$. In this case, during the first $30 \mathrm{~min}$ the shear wave has two ion accumulation nodes, close to $118 \mathrm{~km}$ and $111 \mathrm{~km}$ (Fig. 2c). Within this time interval a sporadic $\mathrm{E}$ layer forms with two maxima of electron density at these ion convergence heights (Fig. 2d). As shown by Shalimov and Haldoupis (2002), the northwestward direction for the horizontal wind turns out to be more efficient in forming a sporadic $\mathrm{E}$ layer.

For the chosen parameters of a shear wave and the direction of the background wind at about $104 \mathrm{~km}$ (Fig. 2c), there exists an ion convergence point, but in this case there was 

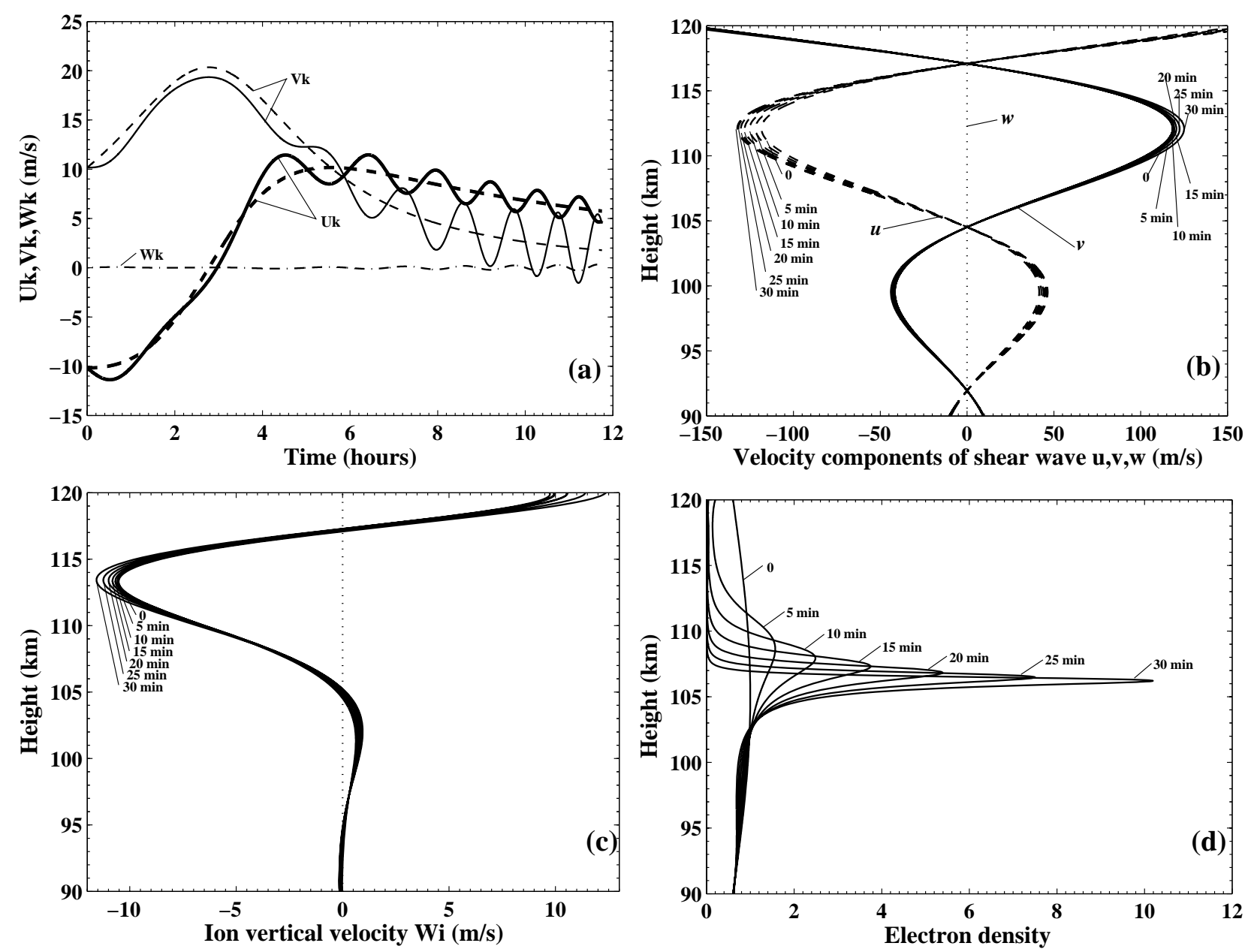

Fig. 1. (a) Temporal development of the shear wave velocity spatial amplitudes (at $\left.h=h_{0}=82 \mathrm{~km}\right) U_{k}(t)$ dark dashed line - analytical approach, dark solid line - numerical simulation), $V_{k}(t)$ (dashed line - analytical approach, solid line - numerical simulation) and $W_{k}(t)$ (dash-dotted line), (b) height variations of horizontal $u$ ( $h, t)$ (dashed line), $v(h, t)$ (solid line) and vertical $w(h, t)$ (dotted line) components of shear wave velocity, (c) height variations of the ion vertical drift velocity $w_{i}(h, t)$ and (d) the formation of a sporadic $E$ layer (for electron density) for time intervals: $t=0,5,10,15,20,25$ and $30 \mathrm{~min}$ in the horizontal shear flow. The linear shear $a=10^{-4} \mathrm{~s}-1$ and the shear wave horizontal and vertical wavelengths $\lambda_{x}=\lambda_{y}=754 \mathrm{~km}$ and $\lambda_{z}=25 \mathrm{~km}$, respectively.

no important increase in electron density near this height (Fig. 2d). This is caused by the smaller value of the shear wave velocity (Fig. 2b) and the increase in the value of the ion-neutral collision frequency at lower heights, which led to smaller values in ion vertical drift velocity (Fig. 2c) $w_{i}$ and a delay in ion convergence (Fig. 2d).

In Figs. 1 and 2 the descending motion of ion accumulation heights and the corresponding downward motion of sporadic E layers are also marked. The velocity is about $5 \mathrm{~km} / \mathrm{h}$ for a single-peaked $E_{s}$ layer (Fig. 1d), and about $1.1 \mathrm{~km} / \mathrm{h}$ for the upper peak and $0.4 \mathrm{~km} / \mathrm{h}$ for the lower peak layers in Fig. 2d. Similar vertical motions and descending speeds of $E_{s}$ layers are known to have been observed elsewhere (e.g. see Mathews, 1998; Haldoupis et al., 2006; Haldoupis and Pancheva, 2006). In the case shown in Fig. 2, the smaller descending velocity for the lower peak, is due to the increase of the ion-neutral collision frequency at the lower height, for which the effect of the shear wave velocity on the ion vertical drift velocity $w_{i}$, Eq. (3), gives a comparatively smaller value in the downward velocity for the lower peak.

Figure 2a shows that the numerical solution of the shear wave (for a smaller vertical wavelength $\lambda_{z}=8 \mathrm{~km}$ ) gives AGW-like oscillations (with a period given by Eq. 16) in its velocity SFH amplitudes, which are more remarkable than those in Fig. 1a shown for a larger vertical wavelength of $25 \mathrm{~km}\left(\lambda_{z}=25 \mathrm{~km}\right)$ (e.g. see Didebulidze et al., 2004). The appearance of AGW-like oscillations in the amplitudes of shear wave velocity SFH is more noticeable for times greater than those necessary for the formation of $E_{s}$ layers ( $t>30 \mathrm{~min}$ ) (see Figs. 1a and 2a). The AGWs can cause 

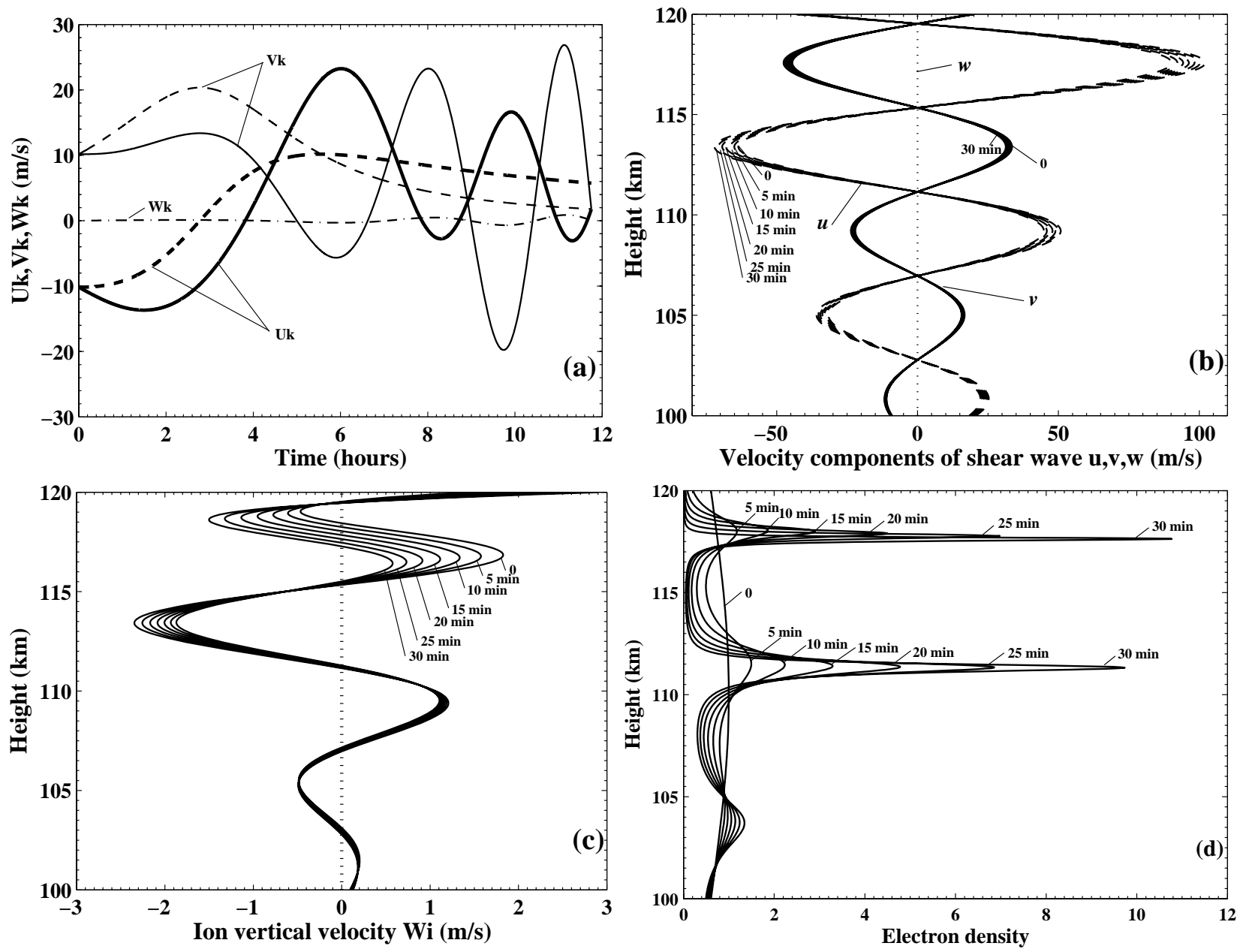

Fig. 2. (a) Temporal development (at $h=h_{0}=94 \mathrm{~km}$ ) of the shear wave velocity spatial amplitudes $U_{k}(t)$ (dark dashed line - analytical approach, dark solid line - numerical simulation), $V_{k}(t)$ (dashed line - analytical approach, solid line - numerical simulation) and $W_{k}(t)$ (dash-dotted line), (b) height variations of horizontal $u(h, t)$ (dashed line), $v(h, t)$ (solid line) and vertical $w(h, t)$ (dotted line) components, (c) height variations of the ion vertical drift velocity $w_{i}(h, t)$ and (d) the formation of a sporadic $E$ layer (for electron density) for time intervals: $t=0,5,10,15,20,25$ and $30 \mathrm{~min}$ in the horizontal shear flow. The shear wave vertical wavelength $\lambda_{z}=8 \mathrm{~km}$ and the other parameters are the same as in Fig. 1.

either compression or rarefaction near the convergence point and the $E_{s}$ layer density decreases with increasing neutral density and vice versa. If the lifetime of sporadic E layers is greater than the characteristic time $t_{a}$ of existence of shear waves, then its density modulation by a AGWs should be important for $t>t_{a}$ and for smaller $t_{a}$, which occurs for greater shear values $a$ of the horizontal wind of Eq. (4), for the same shear wave wavelengths. The presence of oscillation frequencies characteristic of AGWs in sporadic E layers was noticed by several authors (Turunen et al., 1993; Bourdillon et al., 1997; Mathews, 1998; Yokoyama et al., 2004). In our consideration the short-period AGWs have an influence only on ion density variations in the considered regions of the lower thermosphere and do not produce sporadic E layers, including multi-layered structures.
In the cases considered in Figs. 1 and 2 the formation of sporadic E layers occurred for times (about $30 \mathrm{~min}$ ) smaller than the characteristic lifetime $t_{a}$, of a shear wave in the horizontal shear flow. This means that the formation of sporadic E layers is possible for $a>0.0001 \mathrm{~s}^{-1}$ and for different horizontal wavelengths, for which $t_{a}$ is smaller. We also note that at some initial time $t=t_{0}$ when the atmospheric condition is convenient for shear wave excitation, the formation of $\mathrm{E}$ layers in a horizontal shear flow of Eq. (4) for $t-t_{0}<t_{a}-t_{0}$ is indeed possible. 


\section{Discussion}

Several features of sporadic $E$ layers indicate the possibility of their formation under the action of a horizontal wind shear flow that excites a vortical perturbation. This threedimensional incompressible vortical perturbation provides a vertical shear in the neutral horizontal wind which causes, by the combined action of the ion-neutral collisional coupling and the geomagnetic Lorentz forcing, heavy metallic ions $(\mathrm{Fe}+)$ to move vertically and accumulate into thin and dense layers. In the cases investigated in this work, which occur in a horizontal shear flow described by Eq. (4), the formation of sporadic E layers as well as their multi-layer structure and coupling with AGWs are shown to be possible. Such in situ excitation of atmospheric shear waves depends on the initial atmospheric conditions, which are expected to be sporadic for the lower thermosphere and at the different regions of the Earth. The background horizontal wind and the horizontal shear flows, entering Eq. (4), are likely to have different sizes, including that of a regional scale (much lesser than the Earth's radius), which in turn can explain the presence of sporadic E layers on different horizontal scales.

The vertical shears in the tidal wind, which are routinely present in the lower thermosphere are considered as the most important phenomena prerequisite for formation of sporadic E layers (Mathews, 1998; Haldoupis et al., 2006). The tidal winds have horizontal scales much greater than horizontal wavelengths considered for shear excited atmospheric waves. In our model it is assumed that a vertical shear of the background horizontal wind is negligible and the neutral wind height variations are caused by the presence of atmospheric shear waves. The case where the vertical shear in the background horizontal wind is of the same order of scale size (or smaller) as the vertical wavelength of atmospheric waves excited in a horizontal shear flow is not considered here and needs further investigation.

The atmospheric scale height $H$ is assumed constant for the lower thermosphere heights considered in the present study. Height variations of temperature and $H$ can not affect severely the shear parameters of the vortical perturbations.

The horizontal shear flow (4) itself can affect the horizontal motions of the charged particles, as in the mechanism suggested by Shalimov and Haldoupis (2002) for planetary waves. Similar to this mechanism, the horizontal shear flow in Eq. (4), where the horizontal wind velocity $\left(\boldsymbol{u}_{0}\right)$ changes its direction, and the action of the horizontal Lorentz force component can force the ions inwards in regions of a horizontal shear flow with $a<0$ (cyclonic shear) and outwards it $a>0$ (anticyclonic shear), resulting to ion convergence or divergence, respectively. Such horizontal transport of ions and their accumulation inside a horizontal shear flow should give us favourable conditions for the formation of sporadic $\mathrm{E}$ layers in the presence of a vertical windshear with the proper polarity (e.g. see Whitehead, 1989, and references therein). Such horizontal transport of ions during times of sporadic $\mathrm{E}$ formation, as in Figs. 1 and 2, is less effective for the considered shear value in horizontal wind $\left(a^{2} \ll \omega_{b}^{2}\right)$ and for wavelengths $\lambda_{x}, \lambda_{y} \gg \lambda_{z}$. The horizontal transport of ions can become important with increase of the shear, for which shear waves quickly transform into AGWs, which in turn drive the horizontal transport of ions (Chimonas, 1971) thus may affect the Es layer formation process. However, these processes, which are minor compared to those in relation with the atmospheric wave parameters in a horizontal shear flow, were not given a detailed consideration in the present study.

\section{Conclusion}

The formation of mid-latitude sporadic E layers $\left(E_{s}\right.$ layers) in a horizontal shear flow was studied. In a horizontal shear flow, an evolving three-dimensional vortical perturbation (shear wave), whose velocity vector is mainly in the horizontal plane and whose vertical wavenumber $k_{z} \neq 0$, can provide a vertical shear of the horizontal wind. In this case, the effect of shear waves on the vertical transport of heavy metallic ions and their convergence into thin horizontal layers is similar to the "vertical windshear" mechanism of sporadic E layer formation.

The proposed mechanism gives the possibility to explain observed phenomena of a multi-layered structure in the sporadic $\mathrm{E}$ and their different descent speed, which is controlled by the vertical wavelength of the shear wave, the ion-neutral collision frequency and the direction of the background horizontal wind. The formation of single-peaked and multilayered $E_{S}$, which have different descent speeds, was demonstrated numerically for heights in the lower thermosphere.

The property of a shear wave to transform into shortperiod AGWs, the existence of which is also observed in the lower thermosphere, underlines its importance on the variability of sporadic E electron densities. Detailed study of this phenomenon will be the subject of future investigation.

Acknowledgements. This work has been supported by the GNSF/ST07/5-208, INTAS 03-51-6425 and ISTC G-1074 grants.

Topical Editor M. Pinnock thanks two anonymous referees for their help in evaluating this paper.

\section{References}

Banks, P. M. and Kockarts, G.: Aeronomy. Part A, Academic Press inc., New York, NY, 1973.

Bourdillon, A., Lefur, E., Haldoupis, C., Le Roux, Y., Ménard, J., and Delloue, J.: Decameter mid-latitude sporadic-E irregularities in relation with gravity waves, Ann. Geophys., 15, 925-934, 1997 ,

http://www.ann-geophys.net/15/925/1997/.

Chimonas, G.: Enhancement of sporadic-E by horizontal transport within the layer, J. Geophys. Res., 76, 4578-4586, 1971.

Damtie, B., Nygrén, T., Lehtinen, M. S., and Huuskonen, A.: High resolution observations of sporadic-E layers within the polar 
cap ionosphere using a new incoherent scatter radar experiment, Ann. Geophys., 20, 1429-1438, 2002,

http://www.ann-geophys.net/20/1429/2002/.

Didebulidze, G. G.: Evolution of Atmospheric Gravity Waves in Horizontal Shear Flow, Phys. Scripta, 60, 593-600, 1999.

Didebulidze, G. G., Kafkalidis, J. F., and Pataraya, A. D.: Coupling processes between atmospheric vortical perturbations and acustic-gravity waves in the mesosphere-thermosphere regions, J. Atmos. Solar-Terr. Phys., 66, 715-732, 2004.

Gossard, E. E. and Hooke, W. H.: Waves in the atmosphere, Elsevier, Amsterdam, Oxford, New York, 1975.

Haldoupis, C. and Pancheva, D.: Planetary waves and midlatitude sporadic E-layers. Strong experimental evidence for a close relationship, J. Geophys. Res., 107(A6), 1078, doi:10.1029/2001JA000212, 2002.

Haldoupis, C. and Pancheva, D.: Terdiurnal tidelike variability in sporadic E layers, J. Geophys. Res., 111, A07303, doi:10.1029/2005JA011522, 2006.

Haldoupis, C., Meek, C., Christakis, N., Pancheva, D., and Bourdillon, A.: Ionogram height-time-intensity observations of descending sporadic E layers at mid-latitude, J. Atmos. Solar-Terr. Phys., 68, 539-557, 2006.

Hedin, A. E.: Extension of the MSIS thermosphere model into the middle and lower atmosphere. J. Geophys. Res. S. P., 96, 11591172, 1991.

Hines, C. O.: Internal atmospheric gravity waves at ionospheric heights, Can. J. Phys., 38, 1441-1481, 1960.

Hocke, K., Igarashi, K., Nakamura, M., Wilkinson, P., Wu, J., Pavelyev, A., and Wickert, J.: Global sounding of sporadic E layers by the GPS/MET radio occultation experiment, J. Atmos. SolarTerr. Phys., 63, 1973-1980, 2001.

Hussey, G. C., Delloue, J., Haldoupis, C., and Bourdillon, A.: E-region mid-latitude decametre irregularities observed at four radar frequencies. Experiment and first results, Ann. Geophys., 15, 918-924, 1997, http://www.ann-geophys.net/15/918/1997/.

Kelley, M. C., Riggin, D., Pfaff, R. F., Swartz,W. E., Providakes, J. F., and Huang, C.-S.: Large amplitude quasi-periodic fluctuations associated with a mid-latitude sporadic E-layer, J. Atmos. Terr. Phys., 57, 1165-1178, 1995.
Larsen, M. F., Yamamoto,M., Fukao, S., Tsunoda, R. T., and Saito, A.: Observations of neutral winds, wind shears, and wave structure during a sporadic-E/QP event, Ann. Geophys., 23, 23692375, 2005, http://www.ann-geophys.net/23/2369/2005/.

MacLeod, M. A.: Sporadic E Theory. I. Collision-Geomagnetic Equilibrium, J. Atmos. Sci., 23, 96-109, 1966.

Mathews, J. D., Morton, Y. T., and Zhou, Q.: Observations of ion layer motions during the AIDA campaign, J. Atmos. Terr. Phys., 55(3), 447-457, 1993.

Mathews, J. D.: Sporadic E: current views and recent progress, J. Atmos. Solar-Terr. Phys., 60, 413-435, 1998.

Pancheva, D., Haldoupis, C., Meek, C. E., Manson, A. H., and Mitchell, N. J.: Evidence of a role for modulated atmospheric tides in the dependence of sporadic E on planetary waves, J. Geophys. Res., 108(A5), 1176, doi:10.1029/2002JA009788, 2003.

Shalimov, S. and Haldoupis, C.: A model of mid-latitude Eregion plasma convergence inside aplanetary wave cyclonic vortex, Ann. Geophys., 20, 1193-1201, 2002,

http://www.ann-geophys.net/20/1193/2002/.

Turunen, T., Nygrén, T., and Huuskonen, A.: Nocturnal highlatitude E-region in winter during extremely quiet conditions, J. Atmos. Terr. Phys., 55, 783-795, 1993.

Wakabayashi, M. and Ono, T.: Multi-layer structure of mid-latitude sporadic-E observed during the SEEK-2 campaign, Ann. Geophys., 23, 2347-2355, 2005, http://www.ann-geophys.net/23/2347/2005/.

Whitehead, J. D.: Recent work on mid-latitude and equatorial sporadic-E, J. Atmos. Terr. Phys., 51, 401-424, 1989.

Yokoyama, T., Horinouchi, T., Yamamoto, M., and Fukao, S.: Modulation of the mid-latitude ionospheric E-region by atmospheric gravity waves through polarization electric field J. Geophys. Res., 109, A12307, doi:10.1029/2004JA010508, 2004.

Yokoyama, T., Yamamoto, M., and Fukao, S.: Computer simulation of polarization electric fields as a source of mid-latitude field-aligned irregularities, J. Geophys. Res., 108(A2), 1054, doi:10.1029/2002JA009513, 2003.

Yokoyama, T., Yamamoto, M., Fukao, S., Takahashi, T., and Tanaka, M.: Numerical simulation of mid-latitude ionospheric E-region based on SEEK and SEEK-2 observations, Ann Geophys., 23, 2377-2384. 2005. 